\title{
Motor Cortex and Spinal Degeneration in Multisystem Atrophy: A Multimodal Study
}

\author{
Taim Muayqil, K. Ming Chan, Richard Camicioli, Chris Hanstock, John Wong, Luis Concha, \\ Christian Beaulieu, Wendy Johnston, Paul Stenerson, Sanjay Kalra
}

Can. J. Neurol. Sci. 2008; 35: 658-660

Multiple system atrophy (MSA) is a sporadic neuorodegenerative disorder of unknown etiology. A confident diagnosis can be difficult to achieve owing to the varied initial presentation that includes dysautonomia, parkinsonism, cerebellar ataxia and pyramidal signs. ${ }^{1}$ In the absence of a specific test the diagnosis is made largely on clinical grounds. This is unsatisfactory, as a firm diagnosis often cannot be established until the symptoms become fully expressed and the disease has reached an advanced stage. More sensitive measures that can detect sub-clinical manifestations of MSA might be helpful in differentiating it from alternative diagnoses which are attended by different therapeutic and prognostic implications. In this study, we report clinical and pathogenic insights gleaned from the use of novel investigative tools applied to a patient ultimately diagnosed with MSA.

A 50-year-old right handed woman presented with six months of progressive gait impairment and poor balance. For 18 months she had a sensation of "hanging" in her left arm and difficulty manipulating utensils. She denied bulbar, ocular, sensory, and postural symptoms.

Her past medical history was remarkable for a distant left occipital arteriovenous malformation resection that left her with a right visual field defect, and urinary urgency for three years attributed to detrusor instability. The family history was unremarkable.

Examination revealed normal supine and postural blood pressure, a right visual field cut and a mild dysarthria. A spastic catch, worse on the left, was present in all limbs. There was no bradykinesia. Muscle bulk was preserved and there were no fasciculations. Finger and foot tapping were slowed on the left. Strength was decreased in the left finger extensors, hip flexors, knee flexors and extensors at 4/5. Reflexes were brisk bilaterally in upper and lower extremities, with increased activity on the left. The jaw jerk was brisk. A Hoffman's sign was present bilaterally. Ankle clonus was sustained on the left and lasted for eight beats on the right. The plantar response was extensor on the left and flexor on the right. Sensory and cerebellar exams were normal. When walking she had a left foot drop with circumduction.

Blood investigations were all normal, including CBC, urea, electrolytes, thyroid stimulating hormone, B12, calcium, antinuclear antibody, rheumatoid factor, serum protein electrophoresis, angiotensin converting enzyme, paraneoplastic antibodies (anti-Hu, anti-Ri, anti-Yo), human T-lymphotropic virus (HTLV) 1 \& 2, HIV, and lyme serology. Cerebrospinal fluid (CSF) analysis revealed normal cell count, protein and glucose, electrophoresis pattern, and cytologic evaluation. Motor and sensory nerve conduction studies and needle electromyography (EMG) were normal. Conventional magnetic resonance (MR) imaging of the brain revealed only the chronic changes from the resected right occipital arteriovenous malformation with no apparent cortical, cerebellar or brainstem atrophy. An MRI of the entire spinal cord was normal.

Based on this clinical information, primary lateral sclerosis was the provisional diagnosis given the slow progression, prominent diffuse upper motor neuron (UMN) features and absent clinical or EMG evidence of lower motor neuron (LMN) involvement. However, this diagnosis could not be entertained with certainty given that the symptoms duration was under the proposed criteria of three to four years. ${ }^{2,3}$ Thus, she underwent further investigations to explore the extent and time course of degeneration that may shed light on alternative diagnoses (Table).

We found a reduction of motor unit numbers in the lower limb using the multiple point stimulation technique of motor unit number estimation (MUNE).

High field magnetic resonance spectroscopy (MRS) (Figure) was performed as previously described. ${ }^{4}$ The neuronal marker $\mathrm{N}$ acetylaspartate (NAA) (normalized to creatine $(\mathrm{Cr})$, choline (Cho), or myo-inositol (Ins)) was abnormally reduced in the right motor cortex; NAA/Ins was the most abnormal with a reduction of 2.5 standard deviations from the control mean.

Diffusion tensor imaging (DTI) (Figure 1) of the corticospinal tract was performed as previously described..$^{5}$ The corticospinal tract was abnormal at several levels. In the left cerebral peduncle fractional anisotropy (FA) was reduced by 2.1 standard deviations. Bilaterally in the corona radiata trace apparent diffusion coefficient (ADC) was increased by more than 2.5 standard deviations. Trace apparent diffusion coefficient was increased to a moderate degree in the cerebral peduncle

\footnotetext{
From the Division of Neurology (TM), Division of Neurology, Department of Medicine (RC, WJ, SK), Division of Physical Medicine and Rehabilitation/Centre for Neuroscience (KMC), Department of Biomedical Engineering (CH, LC, CB), Faculty of Medicine (JW), University of Alberta (PS), Edmonton, Alberta, Canada. Received January 29, 2008. Final Revisions Submitted June 3, 2008. Correspondence to: Sanjay Kalra, 2E3.18 Walter Mackenzie Health Sciences Centre, University of Alberta Hopsital, 8440-112 Street, Edmonton, Alberta, T6G 2B7, Canada
} 


\section{Table: Results}

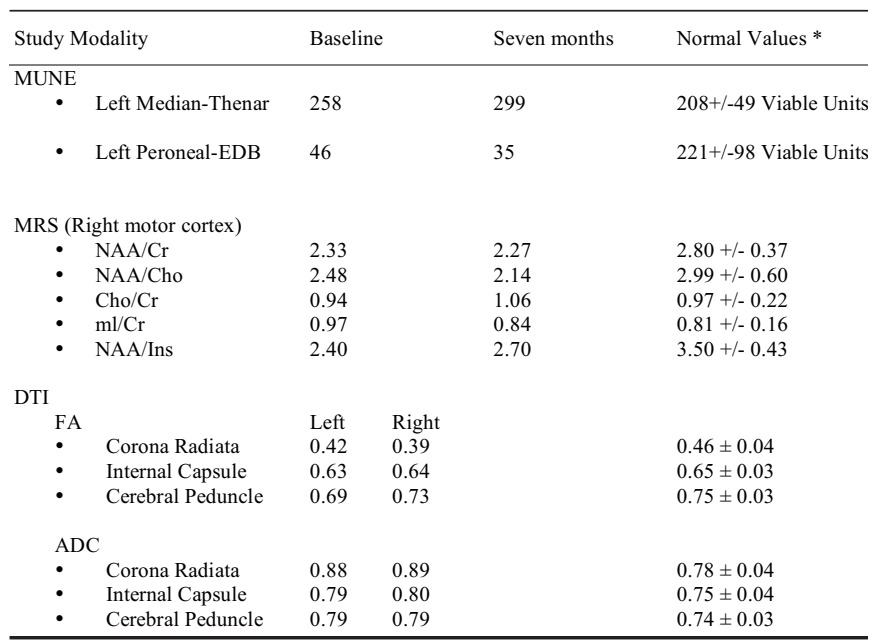

* Normal control data derived from 15 subjects for $\mathrm{MRS}^{4}$ and 15 subjects for $\mathrm{DTI}^{5}$

bilaterally (1.7 standard deviations). Diffusion tensor imaging parameters were normal in the internal capsule.

Paired pulse transcranial magnetic stimulation (TMS) with an inter-pulse interval of $2 \mathrm{~ms}$ was performed with recording of motor evoked potentials (MEP) from the left first dorsal interosseous and extensor digitorum brevis muscles. In healthy individuals, this results in inhibition of at least $30 \%$ (termed 'short interval intracortical inhibition'). However, in this patient, intracortical inhibition was impaired, with only $10 \%$ and $5 \%$ reduction in baseline MEP amplitude respectively (Figure).

Since abnormalities affecting the motor cortex and spinal motor neuron were found, amyotrophic lateral sclerosis (ALS) became a consideration. To investigate this further repeat MUNE and MRS investigations were performed. After seven months, motor unit estimates did not decline further as would have been expected in ALS. ${ }^{6} \mathrm{~N}$-acetylaspartate likely did not change significantly either, given that the interval change in NAA/Cho and NAA/Ins was in opposite directions; with the reciprocal changes of similar magnitude in $\mathrm{Cho} / \mathrm{Cr}$ and $\mathrm{Ins} / \mathrm{Cr}$ this pattern of metabolite changes suggests that Cho and Ins concentration changes had occurred over the observation interval.

\section{Clinical course}

Six months later asymptomatic orthostatic hypotension was documented; seven months later balance further worsened with appearance of hypomimia, bradykinesia and cogwheel rigidity in the left arm. A trial of levodopa-carbidopa provided modest improvement in bradykinesia and gait instability lasting only four months. With the emergence of orthostatic hypotension and extrapyramidal features poorly responsive to levodopa she was diagnosed with probable multiple system atrophy according to
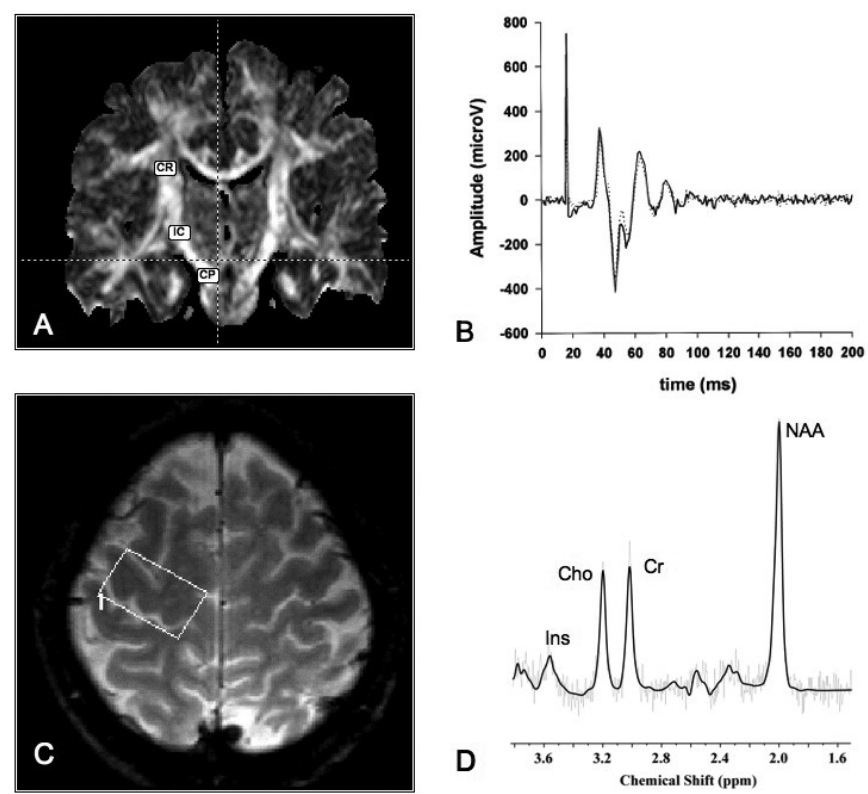

Figure 1. Image A: Coronal diffusion tensor image (FA map) showing regions where diffusion tensor parameters were attained along the corticospinal tract. CR: Corona Radiata; IC: Internal Capsule; CP: Cerebral Peduncle. Image B: Motor evoked potential (MEP) in the extensor digitorum communis muscle following paired pulse transcranial magnetic stimulation with a 2 millisecond interstimulus interval. The baseline MEP is shown in solid line and the response to the paired pulse stimulation in dotted line. In healthy individuals, there should be a $>30 \%$ decrease in the MEP size due to intracortical inhibition. Absence of this decrease in this patient indicates the presence of cortical disinhibition. Image C: Axial gradient-weighted MRI demonstrating voxel placement in the right motor cortex for high-field magnetic resonance spectroscopy. Image D: Corresponding MR spectrum. Ins: myo-Inositol; Cho: choline; Cr: creatine; NAA: $N$-acetylaspartate.

consensus criteria. ${ }^{1}$ After three years of follow up her parkinsonian and autonomic features progressed further and no clinical manifestations of LMN dysfunction nor signs of an alternative neuro-degenerative disorder appeared.

\section{DISCUSSION}

We describe a case of MSA that initially presented with motor neuron disease with restricted UMN signs. Primary lateral sclerosis (PLS) was suspected with motor cortex and corticospinal tract degeneration corroborated by MRS, DTI, and TMS. Standard EMG studies were normal. Although LMN involvement was detected by MUNE raising the suspicion of ALS, this diagnosis was discounted by the absence of progressive decline of motor units or central neuronal degeneration as measured by MRS. The ultimate diagnosis of MSA became apparent when features of autonomic dysfunction and parkinsonism appeared.

Primary lateral sclerosis is a diagnosis of exclusion, obtained after monitoring the course of illness for at least three ${ }^{2}$ to four ${ }^{3}$ 
years without the clinical emergence of lower motor neuron involvement. In PLS, patients complain primarily of stiffness and slowness, and urinary symptoms tend to develop after limb involvement. ${ }^{7}$ The diagnosis of MSA can be difficult in early stages because of its varied presentation. MSA is a sporadic disorder with variable features of dysautonomia, parkinsonism, ataxia and UMN signs. According to consensus criteria, ${ }^{1}$ the diagnostic designations "probable" or "possible" MSA are based on the presence of these clinical features, whereas "definite" MSA requires pathological confirmation. Our patient had the highest clinical designation of probable MSA based on the presence of autonomic failure (orthostatic hypotension) and poorly levodopa-responsive parkinsonism. UMN signs which were very prominent in our patient are actually infrequently observed in MSA, being present in only upwards of $50 \%$ of cases. ${ }^{8}$ Our patient's urinary dysfunction that was attributed to a primary urological condition was perhaps the initial symptom of MSA; urinary dysfunction is the most frequent autonomic disturbance in women with MSA and autonomic dysfunction in general can be a presenting feature in $41 \%$ of cases. ${ }^{9}$

Pathological studies of MSA show prominent involvement of the basal ganglia, cerebellum, pons and intermediolateral cell column, as well as degenerative changes of the corticospinal tract and anterior horn cells. ${ }^{8}$ Our patient presented with the latter as evident clinically and by MRS, DTI and MUNE.

\section{Insights gained through novel investigative techniques}

Advanced and unconventional MRI modalities have been evaluated to assist in distinguishing early MSA from patients with other parkinsonian syndromes. ${ }^{10}$ Magnetic resonance spectroscopy has shown reduced NAA/Cr, a marker of neural integrity, most prominently in the putamen and pons. ${ }^{11-12} \mathrm{We}$ found spectroscopic abnormalities in the motor cortex using NAA and Ins indices. Derangements in Ins in MSA could be reflective of gliosis or abnormal signaling related to the phosphatidylinositol second messenger system. ${ }^{4}$ Progressive cerebral metabolic changes were documented and suggested progression of disease; however, the pattern of longitudinal change in ratios cannot necessarily be ascribed to neuronal dysfunction. A limitation of our MRS protocol was the use of ratios; a study with more subjects would be required with absolute quantification of metabolites to ascertain the changes of specific cerebral chemicals.

Previous DTI studies of MSA have shown reduced FA and increased diffusivity in the basal ganglia, cerebellar peduncle and pons. ${ }^{13-14}$ One study found reduced FA in the internal capsule, ${ }^{15}$ which was not present in this patient. In our DTI protocol that focused on the corticospinal tract, our patient had reduced FA and increased ADC in the corona radiata and cerebral peduncle. TMS revealed prominent cortical disinhibition in keeping with previous studies. ${ }^{16}$

In summary, we describe a patient with a progressive motor neuron disease with prominent UMN signs that was a diagnostic dilemma for several years. Evidence of cerebral degeneration was supported by MRS, DTI, and TMS. The MUNE revealed pronounced subclinical LMN loss. However, the lack of progressive loss of LMNs by MUNE argued against ALS which had important implications with respect to prognosis and treatment. Further study of the biochemical and electro- physiological changes in the brain and spinal cord in patients with MSA could reveal important pathogenic insight in this complex disorder.

\section{ACKNOWLEDGEMENTS}

This study was funded by the University Hospital Foundation. Christian Beaulieu received salary support from AHFMR. Luis Concha received salary support from Promep.

\section{REFERENCES}

1. Gilman S, Low PA, Quinn N, Albanese A, Ben Shlomo Y, Fowler $\mathrm{CJ}$, et al. Consensus statement on the diagnosis of multiple system atrophy. J Neurol Sci. 1999;163(1):94-8.

2. Pringle CE, Hudson AJ, Munoz DG, Kiernan JA, Brown WF, Ebers GC. Primary lateral sclerosis. Clinical features, neuropathology and diagnostic criteria. Brain. 1992;115(Pt 2):495-520.

3. Gordon PH, Cheng B, Katz IB, Pinto M, Hays AP, Mitsumoto H, et al. The natural history of primary lateral sclerosis. Neurology. 2006;66(5):647-53.

4. Kalra S, Hanstock CC, Martin WR, Allen PS, Johnston WS. Detection of cerebral degeneration in amyotrophic lateral sclerosis using high-field magnetic resonance spectroscopy. Arch Neurol. 2006;63(8):1144-8.

5. Wong J, Concha L, Beaulieu C, Johnston W, Allen P, Kalra S. Spatial profiling of the corticospinal tract in amyotrophic lateral sclerosis using diffusion tensor imaging. J Neuroimaging. 2007; 17:234-40.

6. Gooch CL, Shefner JM. ALS surrogate markers. MUNE. Amyotroph Lateral Scler. 2004;5Suppl 1:104-7.

7. Zhai P, Pagan F, Statland J, Butman JA, Floeter MK. Primary lateral sclerosis: a heterogeneous disorder composed of different subtypes? Neurology. 2003;60(8):1258-65.

8. Wenning GK, Tison F, Ben Shlomo Y, Daniel SE, Quinn NP. Multiple system atrophy: a review of 203 pathologically proven cases. Mov Disord. 1997;12(2):133-47.

9. Wenning GK, Ben Shlomo Y, Magalhaes M, Daniel SE, Quinn NP. Clinical features and natural history of multiple system atrophy. An analysis of 100 cases. Brain. 1994;117(Pt 4):835-45.

10. Seppi K, Schocke MF. An update on conventional and advanced magnetic resonance imaging techniques in the differential diagnosis of neurodegenerative parkinsonism. Curr Opin Neurol. 2005;18(4):370-5.

11. Davie CA, Wenning GK, Barker GJ, Tofts PS, Kendall BE, Quinn $\mathrm{N}$, et al. Differentiation of multiple system atrophy from idiopathic Parkinson's disease using proton magnetic resonance spectroscopy. Ann Neurol. 1995;37(2):204-10.

12. Watanabe H, Fukatsu H, Katsuno M, Sugiura M, Hamada K, Okada $\mathrm{Y}$, et al. Multiple regional $1 \mathrm{H}-\mathrm{MR}$ spectroscopy in multiple system atrophy: NAA/Cr reduction in pontine base as a valuable diagnostic marker. J Neurol Neurosurg Psychiatry. 2004;75(1): 103-9.

13. Schocke MF, Seppi K, Esterhammer R, Kremser C, Mair KJ, Czermak BV, et al. Trace of diffusion tensor differentiates the Parkinson variant of multiple system atrophy and Parkinson's disease. Neuroimage. 2004;21(4):1443-1451.

14. Blain CR, Barker GJ, Jarosz JM, Coyle NA, Landau S, Brown RG, et al. Measuring brain stem and cerebellar damage in parkinsonian syndromes using diffusion tensor MRI. Neurology. 2006;67(12):2199-205.

15. Shiga K, Yamada K, Yoshikawa K, Mizuno T, Nishimura $T$, Nakagawa M. Local tissue anisotropy decreases in cerebellopetal fibers and pyramidal tract in multiple system atrophy. $\mathbf{J}$ Neurol. 2005;252(5):589-96.

16. Kuhn AA, Grosse P, Holtz K, Brown P, Meyer BU, Kupsch A. Patterns of abnormal motor cortex excitability in atypical parkinsonian syndromes. Clin Neurophysiol. 2004;115(8): 1786-95. 